\title{
Chemical
} Physics

\section{Dynamic stability ratio of a colloidal dispersion}

\author{
Yung-Chih Kuo, Jyh-Ping Hsu * \\ Department of Chemical Engineering, National Taiwan University, Taipei 10617, Taiwan, ROC
}

\begin{abstract}
The stability ratio of a dispersed system containing colloidal particles having amphoteric surfaces is estimated theoretically. We consider the case where the degree of dissociation of the functional groups on particle surface as a response to the variation in the conditions of the surrounding liquid phase is a function of time. The dynamic nature of the distributions of ions in the electrical double layer near a particle is also taken into account. The result of numerical simulation reveals that the dynamic nature of the system under consideration has the effect of increasing its stability. Due to the fact that the diffusivity of protons in the aqueous solution is much larger than that of other ion species, the level of increase in the stability ratio is less than $\sim 40 \%$. However, if the rates of surface reactions are slow, the dynamic stability ratio can exceeds ten times the corresponding equilibrium value. Also, the smaller the particle and/or the thicker the double layer, the more significant the dynamic behavior of the system under consideration. (C) 1999 Elsevier Science. All rights reserved.
\end{abstract}

Keywords: Stability ratio; Dynamic behavior; Amphoteric surfaces; Double-layer relaxation

\section{Introduction}

One of the basic properties of a colloidal dispersion is its stability ratio, $W$, defined by: [ rate of coagulation between two particles due to pure Brownian motion $) /($ rate of coagulation taking the interac-

\footnotetext{
* Corresponding author. Fax: +886-2-23623040; e-mail: t8504009@ccms.ntu.edu.tw
}

tion energy between two particles into account)]. The numerator is called the rate of rapid coagulation, which can be regarded as the rate of coagulation in the absence of potential barrier, and the denominator is defined as the rate of slow coagulation. The stability ratio measures the effectiveness of potential barrier to prevent particles from coagulation. Classical analysis on the relevant problems was mainly based on specialized conditions on the surfaces of particles, such as constant potential or constant charge density, under a steady state, or time-independent condition. The analysis has been extended to reflect more realistically the actual surface conditions and to include a wider class of colloidal particles in the last two decades. For example, a charge-regulated-surface 
model was proposed by Ninham and Parsegian [1] to simulate the behavior of biological cells. A similar model was adopted by Chang and Hsu [2] to examine the effect of multivalent cations on the rate of adhesion of cells to surfaces. Prieve and Ruckenstein [3] analyzed the interaction between two planar surfaces bearing multiple ionizable functional groups. Chan et al. [4] considered the interaction between two identical amphoteric surfaces under the condition that the electrical free energy is minimized at all distances of separation between two particles; the analysis was also extended to the case of two dissimilar surfaces.

In contrast to the available results for the time-independent stability ratio, the corresponding dynamic cases have been examined to a lesser degree. In the former, the electrical conditions on and near a charged surface reach the equilibrium state instantly as two surfaces approaching each other. As pointed out by Overbeek [5], the relaxation time for surface charges is on the order of $10^{-6}-10^{4} \mathrm{~s}$, and the time scale for Brownian coagulation on the order $10^{-7}$ $10^{-5} \mathrm{~s}$. This implies that the contact between two particles may occur before the electrical conditions reach equilibrium. In a study of the effect of surface-charge relaxation on the interaction between two particles, Kijlstra and van Leeuwen [6] concluded that double-layer dynamics can have a significant influence on the stability ratio. Also, the experimentally observed insensitivity of the stability ratio to particle size may be due to the double-layer dynamics. Lyklema pointed out that, due to the dynamic behavior of double layers [7], the rate of approach of two hydrophobic particles can be reduced significantly [8]. Also the rate of reduction was found to be comparable to that induced by purely hydrodynamic resistance [9]. In a study of the cellular adhesion to a rigid surface, Hsu and Kuo [10] found that the temporal behavior of the dissociation of the ionogenic groups on cell surface has a significant effect on the rate of adhesion. Shulepov et al. [11] investigated the interaction of two colloidal particles by taking the transient behavior of double layer into account. They concluded that the rate constant of the desorption of charge-determining ions plays the major role. Mandralis et al. [12] evaluated the electrical interaction force between two planar, parallel surfaces approaching each other at a constant speed taking the effect of double-layer relaxation into account. The result for two spheres was also derived on the basis of Derjaguin's approximation. The effect of surface charge transport on the stability of spherical colloids was examined by Krozel [13]. It was concluded that the influence of the nonequilibrium charge transport within a particle surface layer is small at low electrical potentials. However, for ion species having a low diffusivity, the correction to the stability ratio needs to be considered. In a study of the dynamic interaction between two electrical double layers, Hsu et al. [14] found that the relaxation of ions in the double layers has the effect of retarding the approaching of two particles.

In a study of the formation of polyanion-polycation complex Koetz et al. [15] found that the colloidal stability of the symplex system is strongly influenced by the variation of charge density on the comonomer units and by that of accessibility of quaternary ammonium functional groups [15]. This experimental observation implies that the classical steady-state analysis can be unrealistic, and an analysis which is capable of taking the dynamic behavior of a colloidal dispersion into account is necessary. This is conducted in the present study.

\section{Analysis}

We consider two charged spherical particles in an $a: b$ electrolyte solution. Let $H_{\mathrm{d}}$ be the closest surface-to-surface distance between two particles. For convenience, the origin of the coordinates is located at one of the end points of the line segment whose length is $H_{\mathrm{d}}$. The surfaces of these particles are charged due to the dissociation of the functional groups they carry, and the degree of dissociation varies with time as a response to the variation in the distance between them. Suppose that the time scales of the coagulation between particles, the dissociation reactions, and the diffusion of ions in the double layer are comparable, that is, their dynamic behaviors needed to be considered simultaneously. For simplicity, we assume that the diffusivities of ions are constant. Also, two particles are sufficiently close 
so that only a one-dimensional problem needs to be considered. In this case, it can be shown that [10]

$$
\begin{gathered}
\frac{\eta}{2} \frac{\mathrm{d} \xi_{\text {con }}}{\mathrm{d} \eta}+\frac{D_{\text {con }}}{D} \frac{\mathrm{d}^{2} \xi_{\text {con }}}{\mathrm{d} \eta^{2}} \\
+\frac{a D_{\text {con }}}{D}\left(\xi_{\text {con }} \frac{\mathrm{d}^{2} \psi}{\mathrm{d} \eta^{2}}+\frac{\mathrm{d} \xi_{\text {con }}}{\mathrm{d} \eta} \frac{\mathrm{d} \psi}{\mathrm{d} \eta}\right)=0, \\
\frac{\eta}{2} \frac{\mathrm{d} \xi_{\text {co }}}{\mathrm{d} \eta}+\frac{D_{\text {co }}}{D} \frac{\mathrm{d}^{2} \xi_{\text {co }}}{\mathrm{d} \eta^{2}} \\
-\frac{b D_{\text {co }}}{D}\left(\xi_{\text {co }} \frac{\mathrm{d}^{2} \psi}{\mathrm{d} \eta^{2}}+\frac{\mathrm{d} \xi_{\text {co }}}{\mathrm{d} \eta} \frac{\mathrm{d} \psi}{\mathrm{d} \eta}\right)=0, \\
\frac{\eta}{2} \frac{\mathrm{d} \xi_{\mathrm{H}^{+}}}{\mathrm{d} \eta}+\frac{D_{\mathrm{H}^{+}}}{D} \frac{\mathrm{d}^{2} \xi_{\mathrm{H}^{+}}}{\mathrm{d} \eta^{2}} \\
+\frac{D_{\mathrm{H}^{+}}}{D}\left(\xi_{\mathrm{H}^{+}} \frac{\mathrm{d}^{2} \psi}{\mathrm{d} \eta^{2}}+\frac{\mathrm{d} \xi_{\mathrm{H}^{+}}}{\mathrm{d} \eta} \frac{\mathrm{d} \psi}{\mathrm{d} \eta}\right)=0, \\
\frac{\mathrm{d}^{2} \psi}{\mathrm{d} \eta^{2}}=\frac{-\nu}{a+b}\left(\xi_{\text {con }}-\xi_{\mathrm{co}}\right) .
\end{gathered}
$$

In these expressions, $\eta=r(D t)^{1 / 2} ; \xi_{\text {con }}=\rho_{\text {con }} / \rho_{0}$; $\xi_{\mathrm{co}}=\rho_{\mathrm{co}} / \rho_{0} ; \varepsilon_{\mathrm{H}^{+}}=\rho_{\mathrm{H}^{+}} / \rho_{0} ; D=\left(D_{\text {con }} D_{\mathrm{co}}\right)^{1 / 2} ; \psi$ $=F \phi / R T ; \quad \nu=D t \kappa ; \quad \kappa^{2}=2 I F^{2} / \varepsilon_{0} \varepsilon_{\mathrm{r}} R T ; \quad \rho_{\mathrm{con}}=$ $a F C_{\mathrm{con}} ; \quad \rho_{\mathrm{co}}=b F C_{\mathrm{co}} ; \quad \rho_{\mathrm{H}^{+}}=F C_{\mathrm{H}^{+}} ; \quad$ and $\rho_{0}=$ $2 I F /(a+b)$. Here, $r$ and $t$ are the position variable and time, respectively; $R, F$, and $T$ are the gas constant, the Faraday constant, and the absolute temperature, respectively; $\phi$ denotes the electrical potential; $\varepsilon_{0}$ and $\varepsilon_{\mathrm{r}}$ are the permittivity of a vacuum and the relative permittivity, respectively; $\rho_{\mathrm{con}}, \rho_{\mathrm{co}}$, and $\rho_{\mathrm{H}^{+}}$are the space charge densities of counter-ions, co-ions, and protons, respectively; $C_{\text {con }}, C_{\mathrm{co}}$, and $C_{\mathrm{H}^{+}}$are the concentrations of counter-ions, co-ions, and protons, respectively; $D_{\text {con }}, D_{\text {co }}$, and $D_{\mathrm{H}^{+}}$are the diffusivities of counter-ions, co-ions, and protons, respectively; $D$ is the geometric mean of $D_{\text {con }}$ and $D_{\text {co }} ; \nu, \kappa$, and $I$ are the scaled time, the reciprocal Debye length, and the ionic strength of the bulk solution ( $\phi \rightarrow 0$ or $r \rightarrow \infty$ ), respectively; $\rho_{0}$ is the counter-ion (or co-ion) charge density in the bulk phase (or as $r \rightarrow \infty$ ); $\xi_{\text {con }}, \xi_{\text {co }}$, and $\varepsilon_{\mathrm{H}^{+}}$are the scaled concentrations of counter-ions, co-ions, and protons, respectively; and $\psi$ represents the scaled electrical potential. The derivation of Eq. (1d) is presented in Appendix A. Here, we assume that the ionic strength is high, and the contribution to the space charge density by both $\mathrm{H}^{+}$and $\mathrm{OH}^{-}$are negligible.

We assume that the surfaces of the particles are ion-impenetrable. Therefore, the boundary conditions associated with Eqs. (1a), (1b), (1c) and (1d) are

$$
\begin{aligned}
& \xi_{\mathrm{con}}=\exp \left(-a \psi_{0}\right), \eta=0 \\
& \xi_{\mathrm{co}}=\exp \left(b \psi_{0}\right), \eta=0 \\
& \frac{\mathrm{d} \xi_{\mathrm{H}^{+}}}{\mathrm{d} \eta}+\xi_{\mathrm{H}^{+}} \frac{\mathrm{d} \psi}{\mathrm{d} \eta}+\frac{a D \eta}{2 D_{\mathrm{H}^{+}} \nu^{1 / 2}} \frac{\mathrm{d} \Sigma^{1}}{\mathrm{~d} \eta}=0, \eta=0
\end{aligned}
$$

$$
\frac{\mathrm{d} \psi}{\mathrm{d} \eta}=\frac{-\Sigma^{1} \nu^{1 / 2}}{(a+b)}, \eta=0,
$$

$\xi_{\text {con }}=\exp \left(-a \psi_{\mathrm{d}}\right), \eta=\frac{H_{\mathrm{d}} \kappa}{\nu^{1 / 2}}$,

$\xi_{\mathrm{co}}=\exp \left(b \psi_{\mathrm{d}}\right), \eta=\frac{H_{\mathrm{d}} \kappa}{\nu^{1 / 2}}$,

$\frac{\mathrm{d} \xi_{\mathrm{H}^{+}}}{\mathrm{d} \eta}+\xi_{\mathrm{H}^{+}} \frac{\mathrm{d} \psi}{\mathrm{d} \eta}-\frac{a D \eta}{2 D_{\mathrm{H}^{+}} \nu^{1 / 2}} \frac{\mathrm{d} \Sigma^{2}}{\mathrm{~d} \eta}=0$,

$\eta=\frac{H_{\mathrm{d}} \kappa}{\nu^{1 / 2}}$,

$$
\frac{\mathrm{d} \psi}{\mathrm{d} \eta}=\frac{\Sigma^{2} \nu^{1 / 2}}{(a+b)}, \eta=\frac{H_{\mathrm{d}} \kappa}{\nu^{1 / 2}} .
$$

In these expressions, $\psi_{0}$ and $\psi_{\mathrm{d}}$ are the scaled electrical potentials at $r=0$ and $r=H_{\mathrm{d}}$, respectively; $\Sigma^{j}=\kappa \sigma^{j} / \rho_{0}\left(\sigma^{j}\right.$ being the surface charge density of particle $j$ ); and $\Sigma^{j}$ the corresponding scaled quantity. The origin of Eqs. (2c) and (2g) is given in Appendix A. Eqs. (2a), (2b), (2e) and (2f) imply that the surfaces of particles are ion-impenetrable. For convenience, it is assumed that the distributions of ions are at equilibrium initially. Therefore, the initial conditions associated with Eqs. (1a), (1b), (1c) and (1d) are

$$
\begin{aligned}
& \xi_{\mathrm{con}}=\xi_{\mathrm{con}}^{\mathrm{e}}=\exp \left(-a \psi^{\mathrm{e}}\right), \eta \rightarrow \infty, \\
& \xi_{\mathrm{co}}=\xi_{\mathrm{co}}^{\mathrm{e}}=\exp \left(b \psi^{\mathrm{e}}\right), \eta \rightarrow \infty, \\
& \xi_{\mathrm{H}^{+}}=\xi_{\mathrm{H}^{+}}^{\mathrm{e}}=\exp \left(-\psi^{\mathrm{e}}\right), \eta \rightarrow \infty,
\end{aligned}
$$

where the superscript e denotes the equilibrium condition. 
Suppose that the functional groups on the surface of a particle are capable of undergoing the following reactions:

$\mathrm{GH}_{2}^{+} \underset{k_{2}}{\stackrel{k_{1}}{\rightleftharpoons}} \mathrm{GH}+\mathrm{H}^{+}$,

$\mathrm{GH} \underset{k_{4}}{\stackrel{k_{3}}{\rightleftharpoons}} \mathrm{G}^{-}+\mathrm{H}^{+}$

where $k_{1}, \ldots, k_{4}$ are rate constants. Suppose that the temporal variations of the concentrations of $\mathrm{GH}_{2}^{+}$ and $\mathrm{G}^{-}$can be described by

$$
\frac{\mathrm{d}\left[\mathrm{GH}_{2}^{+}\right]^{j}}{\mathrm{~d} t}=k_{2}[\mathrm{GH}]^{j} \rho_{\mathrm{H}^{+}, \mathrm{B}}-k_{1}\left[\mathrm{GH}_{2}^{+}\right]^{j},
$$

$\frac{\mathrm{d}\left[\mathrm{G}^{-}\right]^{j}}{\mathrm{~d} t}=k_{3}[\mathrm{GH}]^{j}-k_{4}\left[\mathrm{G}^{-}\right]^{j} \rho_{\mathrm{H}^{+}, \mathrm{B}}$,

where $[\cdot]$ denotes the surface density; and $\rho_{\mathrm{H}^{+}, \mathrm{B}}$ is the value of the space charge density of $\mathrm{H}^{+}, \rho_{\mathrm{H}^{+}}$, on particle surface. Let $K_{1}$ and $K_{2}$ be the equilibrium constants defined as $K_{1}=k_{1} / k_{2}$ and $K_{2}=k_{3} / k_{4}$. The conservation of functional groups gives

$\left[\mathrm{G}^{-}\right]^{j}+[\mathrm{GH}]^{j}+\left[\mathrm{GH}_{2}^{+}\right]^{j}=G^{j}$,

where $G^{j}$ is the total functional groups on particle $j$. We define

$X_{\mathrm{A}}^{j}=1-\left[\mathrm{GH}_{2}^{+}\right]^{j} /\left[\mathrm{GH}_{2}^{+}\right]_{0}^{j}$,

$X_{\mathrm{B}}^{j}=1-\left[\mathrm{G}^{-}\right]^{j} /\left[\mathrm{G}^{-}\right]_{0}^{j}$,

where $\left[\mathrm{GH}_{2}^{+}\right]_{0}^{j}$ and $\left[\mathrm{G}^{-}\right]_{0}^{j}$ are the initial values of $\left[\mathrm{GH}_{2}^{+}\right]^{j}$ and $\left[\mathrm{G}^{-}\right]^{j}$, respectively. Substituting Eqs. (6) and (7a) into Eq. (5a) yields

$$
\begin{aligned}
X_{\mathrm{A}}^{j}= & \frac{M_{2}^{j}}{k_{3}} \frac{\mathrm{d} X_{\mathrm{B}}^{j}}{\mathrm{~d} t}-M_{2}^{j}\left(1+\frac{\rho_{\mathrm{H}^{+}, \mathrm{B}}}{K_{2}}\right) X_{\mathrm{B}}^{j} \\
& +\left(1-M_{1}^{j}+M_{2}^{j}-M_{2}^{j} \frac{\rho_{\mathrm{H}^{+}, \mathrm{B}}}{K_{2}}\right),
\end{aligned}
$$

where $M_{1}^{j}=G^{j} /\left[\mathrm{GH}_{2}^{+}\right]_{0}^{j}, \quad M_{2}^{j}=\left[\mathrm{G}^{-}\right]_{0}^{j} /\left[\mathrm{GH}_{2}^{+}\right]_{0}^{j}$. Substituting Eqs. (6), (7b) and (8) into Eq. (5b), we obtain

$$
\frac{\mathrm{d}^{2} X_{\mathrm{B}}^{j}}{\mathrm{~d} t^{2}}+A^{j} \frac{\mathrm{d} X_{\mathrm{B}}^{j}}{\mathrm{~d} t}+B^{j} X_{\mathrm{B}}^{j}+C^{j}=0,
$$

where

$$
\begin{aligned}
& A^{j}=k_{1}+k_{3}+\left(\frac{k_{1}}{K_{1}}+\frac{k_{3}}{K_{2}}\right) \rho_{\mathrm{H}^{+}, \mathrm{B}}, \\
& B^{j}=k_{1} k_{3}\left(1+\frac{\rho_{\mathrm{H}^{+}, \mathrm{B}}}{K_{2}}+\frac{\rho_{\mathrm{H}^{+}, \mathrm{B}}^{2}}{K_{1} K_{2}}+\frac{1}{k_{1} K_{2}} \frac{\mathrm{d} \rho_{\mathrm{H}^{+}, \mathrm{B}}}{\mathrm{d} t}\right),
\end{aligned}
$$

$C^{j}=k_{1} k_{3} \frac{M_{1}^{j}}{M_{2}^{j}}-B^{j}$.

The initial conditions for Eq. (9) are

$X_{\mathrm{B}}^{j}=0$,

and

$$
\frac{\mathrm{d} X_{\mathrm{B}}^{j}}{\mathrm{~d} t}=\frac{k_{3}}{M_{2}^{j}}\left(1-M_{1}^{j}+M_{2}^{j}-\frac{M_{2}^{j} \rho_{\mathrm{H}^{+}, \mathrm{B}}}{K_{2}}\right) \text { at } t=0 .
$$

If $X_{\mathrm{A}}$ and $X_{\mathrm{B}}$ are evaluated, $\sigma^{j}$ can be estimated by

$$
\begin{aligned}
\sigma^{j} & =e\left(\left[\mathrm{GH}_{2}^{+}\right]^{j}-\left[\mathrm{G}^{-}\right]^{j}\right) \\
& =e G^{j}\left(\frac{1-X_{\mathrm{A}}^{j}}{M_{1}^{j}}-\frac{1-X_{\mathrm{B}}^{j}}{M_{3}^{j}}\right),
\end{aligned}
$$

where $e$ is the elementary charge, and $M_{3}^{j}=M_{1}^{j} / M_{2}^{j}$ $=G^{j} /\left[\mathrm{G}^{-}\right]_{0}^{j}$.

The electrical force per unit area between two parallel plates, $F_{\text {el }}$, can be evaluated by $[14,16]$

$$
\frac{F_{\mathrm{el}}}{C R T}=-\left[\frac{a+b}{2}\left(\frac{\mathrm{d} \psi}{\mathrm{d} X}\right)^{2}+\int_{0}^{\psi}\left(\xi_{\mathrm{con}}-\xi_{\mathrm{co}}\right) \mathrm{d} \psi\right],
$$

where $X=\kappa r$, and $C=a C_{a}^{0}=b C_{b}^{0}$. The electrical potential energy per unit area between two parallel plates, $I_{\mathrm{el}, \mathrm{p}}$, can be calculated by [17]

$I_{\mathrm{el}, \mathrm{p}}=\frac{1}{\kappa} \int_{H^{\prime}}^{\infty} F_{\mathrm{el}}(s) \mathrm{d} s$,

where $H^{\prime}$ is the scaled surface-to-surface distance between two plates; and $s$ is a dummy variable. Applying the Derjaguin approximation, the electrical energy between two spherical particles, $I_{\mathrm{el}}$, can be estimated by

$I_{\mathrm{el}}=2 \pi \int_{0}^{\infty} I_{\mathrm{el}, \mathrm{p}} h \mathrm{~d} h$, 
where $h$ is the radius of interaction ring pairs. Wang et al. [18] suggest using the following approximate expression:

$h \mathrm{~d} h=\frac{1}{\kappa^{2}} \frac{X_{0,1} X_{0,2}}{X_{0,1}+X_{0,2}}\left(1+\frac{1-H}{4 X_{0,1}}+\frac{1-H}{4 X_{0,2}}\right) \mathrm{d} H$,

where $X_{0,1}$ and $X_{0,2}$ are the scaled radii of particles 1 and 2, respectively; and $H$ is the scaled closest surface-to-surface distance between two particles. Substituting Eqs. (12) and (14) into Eq. (13) yields

$$
\begin{aligned}
I_{\mathrm{el}}= & \frac{2 \pi}{\kappa^{3}} \frac{X_{0,1} X_{0,2}}{X_{0,1}+X_{0,2}} \int_{H}^{\infty}\left[\left(1+\frac{1-s}{4 X_{0,1}}+\frac{1-s}{4 X_{0,2}}\right)\right. \\
& \left.\times \int_{s}^{\infty} F_{\mathrm{el}}(u) \mathrm{d} u\right] \mathrm{d} s,
\end{aligned}
$$

where $s$ and $u$ are dummy variables. For identical particles, $X_{0,1}=X_{0,2}=X_{0}$, and Eq. (15) reduces to $I_{\mathrm{el}}=\frac{\pi X_{0}}{\kappa^{3}} \int_{H}^{\infty}\left[\left(1+\frac{1-s}{2 X_{0}}\right) \int_{s}^{\infty} F_{\mathrm{el}}(u) \mathrm{d} u\right] \mathrm{d} s$.

The van der Waals energy between two particles, $I_{\mathrm{vdW}}$, can be evaluated by $[19,20]$

$$
\begin{aligned}
I_{\mathrm{vdW}}= & \frac{-A_{132}}{2 H_{1}}\left\{\frac{1}{1+D_{4} H_{1}}+\frac{H_{1}}{1+H_{1}+D_{4} H_{1}^{2}}\right. \\
& \left.+2 H_{1} \ln \left[\frac{H_{1}+D_{4} H_{1}^{2}}{1+H_{1}+D_{4} H_{1}^{2}}\right]\right\},
\end{aligned}
$$

where $A_{132}$ is the Hamaker constant; $D_{4}=D_{3} / 2 D_{2}$; $D_{3}=D_{1} / D_{2} ; \quad D_{2}=X_{0,1}+X_{0,2} ; \quad D_{1}=2 X_{0,1} X_{0,2}$; and $H_{1}=\left(R-D_{2}\right) / D_{3}$. For identical particles, $X_{0,1}$ $=X_{0,2}=X_{0}$, and Eq. (16) becomes

$$
\begin{aligned}
I_{\mathrm{vdW}}= & \frac{-A_{132}}{3 H_{1}}\left\{\frac{1}{4+H_{1}}+\frac{H_{1}}{4+4 H_{1}+H_{1}^{2}}\right. \\
& \left.+\frac{H_{1}}{2} \ln \left[\frac{4 H_{1}+H_{1}^{2}}{4+4 H_{1}+H_{1}^{2}}\right]\right\} .
\end{aligned}
$$

The hydrodynamic force between two spherical particles, $F_{\mathrm{H}}$, can be evaluated by [21]

$F_{\mathrm{H}}=-6 \pi \mu \beta D_{3} \nu / \kappa$, where $\mu$ is the viscosity of the liquid phase; $\beta$ the retardation factor; and $v$ the relative velocity between two particles. If $X_{0,1}=X_{0,2}=X_{0}$, Eq. (17) reduces to

$F_{\mathrm{H}}=-6 \pi \mu \beta X_{0} \nu / \kappa$,

where

$$
\begin{aligned}
\beta= & \frac{4}{3}[\sinh (\alpha)] \sum_{n=0}^{\infty}\left\{\frac{n(n+1)}{(2 n-1)(2 n+3)}\right. \\
& \left.\times\left[\frac{4 \cosh ^{2}\left[\left(n+\frac{1}{2}\right) \alpha\right]+(2 n+1)^{2} \sinh ^{2}(\alpha)}{2 \sinh [(2 n+1) \alpha]-(2 n+1) \sinh (2 \alpha)}-1\right]\right\},
\end{aligned}
$$

$\alpha=\cosh ^{-1}\left(1+H / X_{0}\right)$.

The hydrodynamic retardation energy can be calculated by

$I_{\mathrm{h}}=\frac{1}{\kappa} \int_{H}^{\infty} F_{\mathrm{H}} \mathrm{d} H$.

The total interaction energy between two particles, $I_{\mathrm{t}}$, comprises of the electrical energy, the van der Waals energy, and the hydrodynamic retardation energy, that is,

$I_{\mathrm{t}}=I_{\mathrm{el}}+I_{\mathrm{vdW}}+I_{\mathrm{h}}$.

Suppose that the effect of random force between two particles can be neglected, that is, only the mean behavior of the system is considered. In this case, it can be shown that the temporal variation of $H$ is described by $[10,14]$

$$
\frac{\mathrm{d}^{2} H}{\mathrm{~d} t^{2}}+\frac{3 \kappa^{5}\left(\rho_{1} X_{0,1}^{3}+\rho_{2} X_{0,2}^{3}\right)}{4 \pi \rho_{1} \rho_{2} X_{0,1}^{3} X_{0,2}^{3}} \frac{\partial I_{\mathrm{t}}}{\partial H}=0,
$$

where $\rho_{1}$ and $\rho_{2}$ are the densities of particles 1 and 2 , respectively. For identical particles, $\rho_{1}=\rho_{2}=\rho$, $X_{0,1}=X_{0,2}=X_{0}$, and Eq. (20) becomes

$$
\frac{\mathrm{d}^{2} H}{\mathrm{~d} t^{2}}+\frac{3 \kappa^{5}}{2 \pi \rho X_{0}^{3}} \frac{\partial I_{\mathrm{t}}}{\partial H}=0 .
$$


The time-averaged stability ratio of the system under consideration, $W$, can be evaluated by (Appendix A)

$W=\frac{1}{t_{\mathrm{s}}} \int_{0}^{t_{\mathrm{s}}} \omega \mathrm{d} t$

where $t_{\mathrm{s}}$ denotes the time of slow flocculation and $\omega$ is the time-dependent stability ratio, which satisfies

$\int_{1}^{\infty} \frac{\exp \left(I_{\mathrm{t}} / k_{\mathrm{B}} T\right)}{R_{\mathrm{r}}^{2} \omega} \mathrm{d} R_{\mathrm{r}}=1$

where $k_{\mathrm{B}}$ is the Boltzmann constant, and $R_{\mathrm{r}}=1+$ $H / D_{2}$. For identical particles, $X_{0,1}=X_{0,2}=X_{0}$, and Eq. (21a) becomes

$$
\int_{0}^{\infty} \frac{2 X_{0}}{\omega\left(2 X_{0}+H\right)^{2}} \exp \left(I_{\mathrm{t}} / k_{\mathrm{B}} T\right) \mathrm{d} H=1 .
$$

\section{Results and discussion}

The behavior of the system under consideration is examined through numerical simulation. We suggest using the procedure below to estimate the stability ratio.

Step (1): At the $k$ th stage, the time is $t_{k}$. The separation distance between two particles is guessed as $H_{k, \mathrm{~g}}$. The conversion $X_{\mathrm{B}, k}^{j}$ is evaluated by solving Eq. (9) subject to Eqs. (9d) and (9e), and the conversion $X_{\mathrm{A}, k}^{j}$ is calculated by Eq. (8), using the value of $\rho_{\mathrm{H}^{+}, \mathrm{B}}$ at the $(k-1)$ th stage, $\rho_{\mathrm{H}^{+}, \mathrm{B}, k-1}$. The surface charge density is estimated by Eq. (10).

Step (2): The electrical potential at the $k$ th stage, $\phi_{k}$, and the space charge density of protons, $\rho_{\mathrm{H}^{+}, k}$, estimated by solving Eqs. (1a), (1b), (1c) and (1d) subject to Eqs. (2a), (2b), (2c), (2d), (2e), (2f), (2g) and (2h). Determine the value of $\rho_{\mathrm{H}^{+}, B, k}$, and return to step (1). This is repeated until a convergent $\rho_{\mathrm{H}^{+}, \mathrm{B}, k}$ is obtained.

Step (3): $F_{\mathrm{el}, k}, I_{\mathrm{el}, k}$, and $I_{\mathrm{vdW}, k}$ are calculated by Eqs. (11), (15) and (16), respectively.

Step (4): $F_{\mathrm{H}, k}$ and $I_{\mathrm{h}, k}$ are evaluated by Eqs. (17) and (18) respectively, using the $v$ at the previous stage, $v_{k-1}$.
Step (5): $I_{\mathrm{t}}$ is calculated by Eq. (19). Solving Eq. (20) subject to the conditions $H=H_{k-1}$, and $v=$ $v_{k-1}$ at $t=t_{k-1}$, the value of $H$ at $t_{k}, H_{k}$, and that of $v$ at $t_{k}, v_{k}$, are obtained. Return to step (4) with the modified $v_{k}$. This is repeated until $v_{k}$ converges.

Step (6): If $H_{k, \mathrm{~g}}$ and $H_{k}$ are inconsistent, the former is replaced by the latter and return to step (1). This is repeated until a convergent $H_{k}$ is obtained.

Step (7): The time is advanced by $t_{k+1}=t_{k}+\Delta t$, $\Delta t$ being a pre-specified time interval, and return to step (1).

Step (8): $W$ is estimated by Eq. (21).

Fig. 1 shows the variation of the stability ratio as a function of the linear size of a particle for various density of the functional groups on the surfaces of particles. The results based on the equilibrium model in which the dynamic behavior of double-layer relaxation and that of the dissociation of functional groups are neglected, are also shown for comparison. At

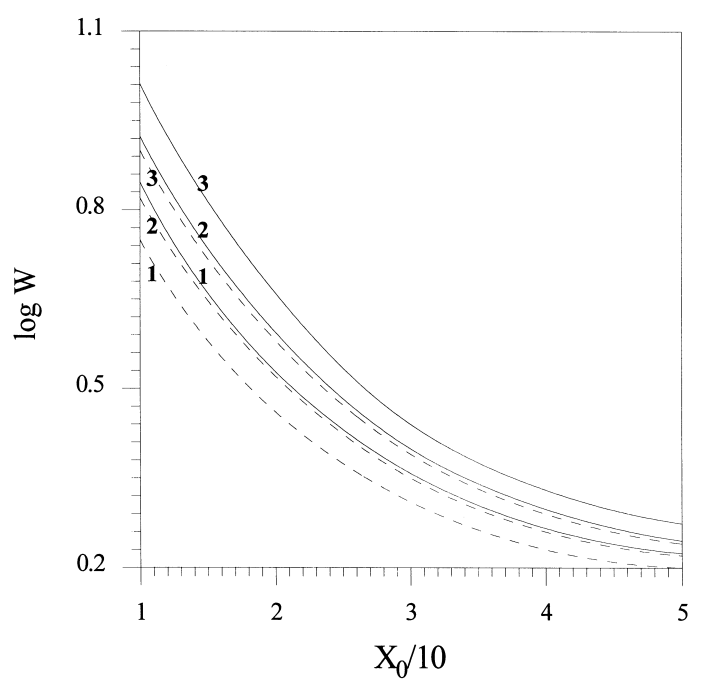

Fig. 1. Variation of the stability ratio as a function of the linear size of a particle for the case $I=1 \times 10^{-3} \mathrm{M}, A_{132}=10^{-21} \mathrm{~J}$. Key: $a=2, b=1, \varepsilon_{\mathrm{r}}=78, T=298.15 \mathrm{~K}, k_{1}=k_{3}=1.5 \times 10^{4}$ $\mathrm{s}^{-1}, K_{1}=K_{2}=4 \times 10^{-5} \mathrm{M}, M_{1}=10, M_{2}=20, G^{1}=G^{2}=G$, $X_{0,1}=X_{0,2}=X_{0}, \rho_{1}=\rho_{2}=\rho=10^{3} \mathrm{~kg} / \mathrm{m}^{3}, \mathrm{pH}=7, \mu=10^{-3}$ Pas, $D_{\text {con }}=D_{\text {co }}=10^{-9} \mathrm{~m}^{2} / \mathrm{s}, D_{\mathrm{H}^{+}}=9.312 \times 10^{-9} \mathrm{~m}^{2} / \mathrm{s}$. The initial conditions are $v=0 \mathrm{~m} / \mathrm{s}, H=X_{0} / 10$. The solid line represents the result predicted by the present dynamic model, and the dash line denotes those predicted by the corresponding equilibrium model. Curves 1: $G=5 \times 10^{16}$ groups $/ \mathrm{m}^{2} ; 2: G=7 \times 10^{16}$ groups $/ \mathrm{m}^{2}, 3: G=10^{17}$ groups $/ \mathrm{m}^{2}$. 
equilibrium, the Poisson equation, Eq. (1d), reduces to

$$
\frac{\mathrm{d}^{2} \psi}{\mathrm{d} X^{2}}=\frac{g}{a+b},
$$

where $g=\exp (b \psi)-\exp (-a \psi)$. The boundary conditions associated with Eq. (22) are (Appendix A)

$$
\begin{aligned}
\frac{\mathrm{d} \psi}{\mathrm{d} X}= & \frac{-\kappa G^{1}}{(a+b) C N_{\mathrm{A}}}\left(\frac{\rho_{\mathrm{H}^{+}, \mathrm{B}}^{\mathrm{e}}}{K_{1}}-\frac{K_{2}}{\rho_{\mathrm{H}^{+}, \mathrm{B}}^{\mathrm{e}}}\right) \\
& \times\left(\frac{\rho_{\mathrm{H}^{+}, \mathrm{B}}^{\mathrm{e}}}{K_{1}}-\frac{K_{2}}{\rho_{\mathrm{H}^{+}, \mathrm{B}}^{\mathrm{e}}}+1\right)^{-1}
\end{aligned}
$$

at $X=0$,

$$
\begin{aligned}
\frac{\mathrm{d} \psi}{\mathrm{d} X}= & \frac{-\kappa G^{2}}{(a+b) C N_{\mathrm{A}}}\left(\frac{\rho_{\mathrm{H}^{+}, \mathrm{B}}^{\mathrm{e}}}{K_{1}}-\frac{K_{2}}{\rho_{\mathrm{H}^{+}, \mathrm{B}}^{\mathrm{e}}}\right) \\
& \times\left(\frac{\rho_{\mathrm{H}^{+}, \mathrm{B}}^{\mathrm{e}}}{K_{1}}-\frac{K_{2}}{\rho_{\mathrm{H}^{+}, \mathrm{B}}^{\mathrm{e}}}+1\right)^{-1}
\end{aligned}
$$

at $X=H$,

where $N_{\mathrm{A}}$ is the Avogadro number. Fig. 1 reveals that at a fixed density of the functional groups on surfaces of particles, $G$, the smaller the particles, the larger the stability ratio. This is expected since if $G$ is fixed, the smaller the particles, the smaller the van der Waals attractive energy between two particles. Fig. 1 also suggests that the higher the value of $G$, the greater the stability ratio. This is because that the higher the value of $G$, the greater the electrical repulsive energy between two particles. As can be seen from Fig. 1, the stability ratio based on a dynamic model is greater than that based on the corresponding equilibrium model. That is, the dynamic nature of the system under consideration has the effect of increasing its stability. The deviations of the result based on the equilibrium from that base on the dynamic model are $13.6 \%, 14.5 \%$, and $15.9 \%$ for curves 1,2 , and 3 , respectively. Note that the diffusivity of protons in the aqueous solution is much larger than that of other ion species $\left(D_{\mathrm{H}^{+}} \approx 10 D\right.$ is used in the numerical simulation). It is expected that the spatial distribution of protons will be much closer to the equilibrium Boltzmann distribution than other ion species, as was observed in our previous study [22]. Therefore, if the rates of surface reactions are fast ( $k_{i}$, is large, $\left.i=1, \ldots, 4\right)$, the effect of the dynamic behavior of the system under consideration on the stability ratio becomes inappreciable. The average deviation for $X_{0}=1,3$, and 5 , are $27.2 \%$, $11.9 \%$, and $6.6 \%$, respectively. This implies that the smaller the particle, the more significant the dynamic behavior of system under consideration.

Fig. 2 illustrates the variation of the stability ratio as a function of Debye length, $(1 / \kappa)$, for various Hamaker constants $A_{132}$. The results for the corresponding equilibrium model are also presented in this figure. Fig. 2 reveals that, the larger the $(1 / \kappa)$ (thicker the double layer), or the smaller the $A_{132}$, the larger the $W$, as expected. As in the case of Fig. 1 , the equilibrium model will underestimate the stability ratio. The deviations are $22.1 \%, 23.3 \%$, and $24.7 \%$ for curves 1,2 , and 3 , respectively. The average deviation for $1 / \kappa=10,30$, and 50 are $13.3 \%, 21.7 \%$, and $34.5 \%$, respectively. This implies that the thicker the double layer the more significant the dynamic behavior of the system under consideration.

The information about the magnitudes of the surface rate constants is very limited in the literature. To estimate its effect on the stability ratio, the rate constant for a reaction occurs in bulk phase is adopted as reference. It can assume a wide range of magni-

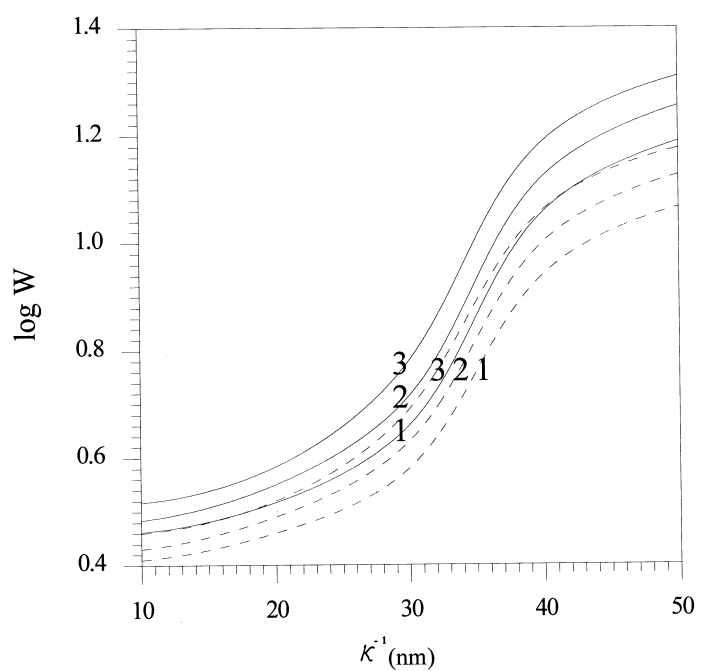

Fig. 2. Variation of the stability ratio as a function of the Debye length $(1 / \kappa)$ for the case $G^{1}=G^{2}=G=7 \times 10^{16}$ groups $/ \mathrm{m}^{2}$, $r_{0}=2.5 \times 10^{-7} \mathrm{~m}$. Key: same as in caption of Fig. 1. Curves 1: $A_{132}=10^{-20} \mathrm{~J} ; 2: A_{132}=10^{-21} \mathrm{~J} ; 3: A_{132}=10^{-22} \mathrm{~J}$. 
tudes. The rate constant for the decomposition of ethylbenzene to yield benzene and ethylene, for example, has the value $2.2 \times 10^{-32} \mathrm{~s}^{-1}$, that for the decomposition of di-t-butyl peroxide to form acetone and ethane is $4.6 \mathrm{~s}^{-1}$, and that for the oxidation of naphthalene over vanadium pentoxide catalyst to produce phthalic anhydride is $5.3 \times 10^{15} \mathrm{~s}^{-1}$ [23]. The simulated variation of the stability ratio as a function of particle size at various rates of surface reactions are illustrated in Fig. 3. This figure suggests that the deviations of the dynamic stability ratio from the corresponding equilibrium values are $13.6 \%, 50.1 \%$, and $298.7 \%$ for curves 1, 2, and 3, respectively. In other words, the slower the surface reactions, the greater the difference between the dynamic stability ratio and the corresponding equilibrium stability ratio, that is, the more significant the dynamic behavior of the system under consideration. It is worth to note that the deviation is $833.2 \%$ at $X_{0}=1$ for the case of curve 3 . This means that the dynamic behavior may lead to about tenfold deviation in the evaluation of the stability ratio. Fig. 3 also reveals that the smaller the particle, the greater the deviation.

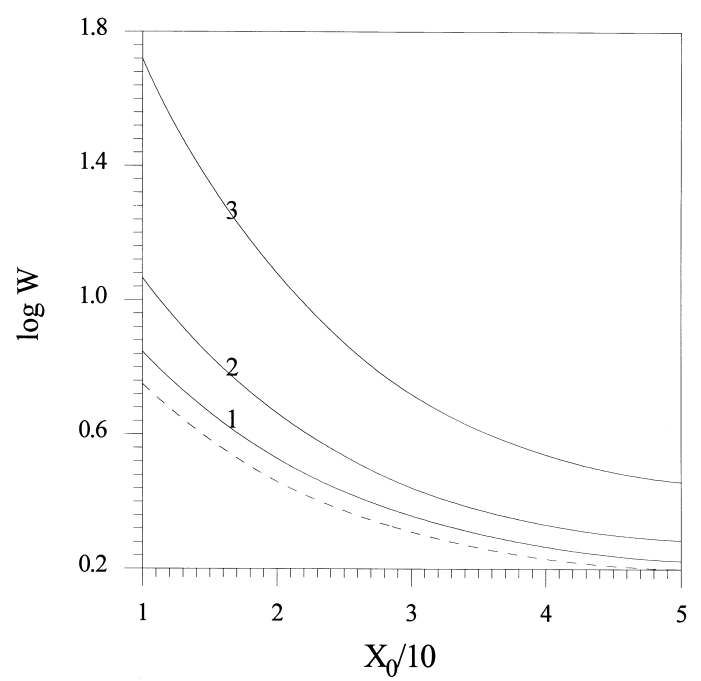

Fig. 3. Variation of the stability ratio as a function of particle size at various rates of surface reactions for the case $I=1 \times 10^{-3} \mathrm{M}$, $A_{132}=10^{-21} \mathrm{~J}, G=5 \times 10^{16}$ groups $/ \mathrm{m}^{2}$. Key: same as in caption of Fig. 1 except that $k_{1}$ and $k_{3}$ are different. Curves 1: $k_{1}=k_{3}=1.5 \times 10^{4} \mathrm{~s}^{-1} ; 2: k_{1}=k_{3}=3 \times 10^{2} \mathrm{~s}^{-1} ; 3: k_{1}=k_{3}=10$ $\mathrm{s}^{-1}$.
Note that under the conditions assumed in Figs. $1-3$, the surfaces of particles are negatively charged. Therefore, the role of $\mathrm{H}^{+}$is more significant than that of $\mathrm{OH}^{-}$. Also, since the ionic strength is relatively high, the contributions to the space charge density in the Poisson Eq. (1d), by both $\mathrm{H}^{+}$and $\mathrm{OH}^{-}$can be neglected. The concentration of $\mathrm{H}^{+}$on particle surface, however, needs to be considered since it is related to the dissociation of the functional groups.

The equilibrium constants for the reactions represented by Eqs. (4a) and (4b) can be expressed by

$K_{1}=[\mathrm{GH}] \rho_{\mathrm{H}^{+}, \mathrm{B}} /\left[\mathrm{GH}_{2}^{+}\right]$,

$K_{1}=\left[\mathrm{G}^{-}\right] \rho_{\mathrm{H}^{+}, \mathrm{B}} /[\mathrm{GH}]$.

If temperature is fixed, both $K_{1}$ and $K_{2}$ are fixed. Therefore, the lower the $\mathrm{pH}$, the higher the $\left[\mathrm{GH}_{2}^{+}\right]$ and lower the $\left[\mathrm{G}^{-}\right]$. In other words, the amount of negative charges on particle surface decreases with a decrease in solution $\mathrm{pH}$. This leads to a smaller electrostatic interaction between two particles, and the dispersion becomes more unstable.

\section{Acknowledgements}

This work is partially financially supported by the National Science Council of the Republic of China.

\section{Appendix A}

The Poisson equation can be written as [24]

$$
\frac{\mathrm{d}^{2} \psi}{\mathrm{d} X^{2}}=\frac{g_{0}}{U},
$$

where

$\kappa^{2}=F^{2} U / \varepsilon_{0} \varepsilon_{\mathrm{r}} R T$,

$g_{0}=C_{\mathrm{OH}^{-}}-C_{\mathrm{H}^{+}}+b C_{b}-a C_{a}$,

$U=a^{2} C_{a}^{0}+b^{2} C_{b}^{0}+C_{\mathrm{H}^{+}}^{0}+C_{\mathrm{OH}^{-}}^{0}$,

where $C_{a}, C_{b}$, and $C_{\mathrm{OH}^{-}}$are the concentrations of cations, anions, and hydroxyl groups, respectively; and the superscript 0 denotes the bulk property. Suppose that the bulk $\mathrm{pH}$ is $7, C_{\mathrm{H}^{+}}^{0} \ll C_{a}^{0}$, and the surface of a particle is not highly charged. In this case, Eqs. (A.1a) and (A.1b) reduce to, respectively, $g_{0}=b C_{b}-a C_{a}$, 
and

$$
U=a(a+b) C_{a}^{0} .
$$

Substituting Eqs. (A.2a) and (A.2b) into Eq. (A.1), Eq. (1d) can be recovered.

If $\mathrm{H}^{+}$is released by the functional groups on the surface of a particle, the surface become negatively charged. This induces an $\mathrm{H}^{+}$flux in the double layer near the surface, $N_{\mathrm{H}^{+}}$. We have

$N_{\mathrm{H}^{+}}=\frac{(-)^{j}}{F} \frac{\mathrm{d} \sigma^{j}}{\mathrm{~d} t}, j=1$ or 2,

where $j$ is an index, $j=2$ represents the surface on the right-hand side, and $j=1$ that on the left-hand side. Employing the Nernst-Planck equation, Eq. (A.3) becomes

$$
\begin{gathered}
\left(\frac{\partial C_{\mathrm{H}^{+}}}{\partial r}\right)_{r=B}+\left(C_{\mathrm{H}^{+}} \frac{\partial \psi}{\partial r}\right)_{r=B} \\
+\frac{(-1)^{j}}{F D_{\mathrm{H}^{+}}} \frac{\mathrm{d} \sigma^{j}}{\mathrm{~d} t}=0,
\end{gathered}
$$

where $B$ denotes the solid-liquid surfaces. This expression can be rewritten in the following scaled form:

$$
\begin{gathered}
\left(\frac{\mathrm{d} \xi_{\mathrm{H}^{+}}}{\mathrm{d} \eta}\right)_{r=B}+\left(\xi_{\mathrm{H}^{+}} \frac{\mathrm{d} \psi}{\mathrm{d} \eta}\right)_{r=B} \\
+\frac{(-1)^{j+1} a D \eta}{2 D_{\mathrm{H}^{+}} \nu^{1 / 2}} \frac{\mathrm{d} \Sigma^{j}}{\mathrm{~d} \eta}=0 .
\end{gathered}
$$

Eqs. (2c) and (2g) can be recovered by this expression.

If the surface reactions described by Eqs. (4a) and (4b) are at equilibrium, then

$$
\begin{aligned}
& K_{1}=\frac{[\mathrm{GH}]^{j, \mathrm{e}} \rho_{\mathrm{H}^{+}, \mathrm{B}}^{\mathrm{e}}}{\left[\mathrm{GH}_{2}^{+}\right]^{j, \mathrm{e}}}, \\
& K_{2}=\frac{[\mathrm{G}-]^{j, \mathrm{e}} \rho_{\mathrm{H}^{+}, \mathrm{B}}^{\mathrm{e}}}{[\mathrm{GH}]^{j, \mathrm{e}}},
\end{aligned}
$$

where the superscript e denotes the equilibrium state. Substituting these expressions into Eq. (10) gives

$$
\sigma^{j}=e[\mathrm{GH}]^{j, \mathrm{e}}\left(\frac{\rho_{\mathrm{H}^{+}, \mathrm{B}}^{\mathrm{e}}}{K_{1}}-\frac{K_{2}}{\rho_{\mathrm{H}^{+}, \mathrm{B}}^{\mathrm{e}}}\right) \text {. }
$$

From Eqs. (A.7) and (6), we have

$$
\begin{aligned}
\sigma^{j}= & e G^{j}\left(\frac{\rho_{\mathrm{H}^{+}, \mathrm{B}}^{\mathrm{e}}}{K_{1}}-\frac{K_{2}}{\rho_{\mathrm{H}^{+}, \mathrm{B}}^{\mathrm{e}}}\right) \\
& \times\left(\frac{\rho_{\mathrm{H}^{+}, \mathrm{B}}^{\mathrm{e}}}{K_{1}}-\frac{K_{2}}{\rho_{\mathrm{H}^{+}, \mathrm{B}}^{\mathrm{e}}}+1\right)^{-1} .
\end{aligned}
$$

Eqs. (2d) and (2h) lead to

$$
\frac{\mathrm{d} \psi}{\mathrm{d} X}=\frac{(-1)^{j} \kappa \sigma^{j}}{(a+b) \rho_{0}} \text {. }
$$

Substituting Eq. (A.8) into Eq. (A.9) yields Eqs. (22a) and (22b).

According to the theory of fast coagulation [19,25],

$\int_{D_{2} / \kappa}^{\infty} \frac{J_{\mathrm{f}}}{r^{2}} \mathrm{~d} r=-4 \pi D_{\text {eff }} C_{0}$,

where $J_{\mathrm{f}}$ is the particle flux in the absence of energy barrier, $D_{\text {eff }}$ is the effective mutual diffusivity of particles, and $C_{0}$ is the bulk particle concentration. At steady state, Eq. (A.10) becomes

$$
\frac{\kappa}{D_{2}} J_{\mathrm{f}}=-4 \pi D_{\mathrm{eff}} C_{0},
$$

In the case of slow coagulation,

$\int_{D_{2} / \kappa}^{\infty} \frac{J_{\mathrm{s}}}{r^{2}}\left(\frac{I_{\mathrm{t}}}{k_{\mathrm{B}} T}\right) \mathrm{d} r=-4 \pi D_{\mathrm{eff}} C_{0}$,

where $J_{\mathrm{s}}$ denotes the particle flux in the presence of energy barrier. If the coagulation between particles can be considered as a second-order process, then Eqs. (A.11) and (A.12) lead to

$$
\frac{\kappa}{D_{2}} J_{\mathrm{f}}=\int_{D_{2} / \kappa}^{\infty} \frac{J_{\mathrm{s}}}{r^{2}} \exp \left(\frac{I_{\mathrm{t}}}{k_{\mathrm{B}} T}\right) \mathrm{d} r .
$$

Eq. (21a) can be recovered from Eq. (A.13).

\section{References}

[1] B.W. Ninham, V.A. Parsegian, J. Theor. Biol. 31 (1971) 405.

[2] Y.I. Chang, J.P. Hsu, J. Theor. Biol. 147 (1990) 509.

[3] D.C. Prieve, E. Ruckenstein, J. Theor. Biol. 56 (1976) 205.

[4] D. Chan, J.W. Perram, L.R. White, T.W. Healy, J. Chem. Soc., Faraday Trans. I 71 (1975) 1046.

[5] J.Th.G. Overbeek, J. Colloid Interface Sci. 58 (1977) 408.

[6] J. Kijlstra, H.P. van Leeuwen, J. Colloid Interface Sci. 160 (1993) 424. 
[7] J. Lyklema, Pure Appl. Chem. 52 (1980) 1221.

[8] J. Lyklema, in: D.M. Bloor, E. Wyn-Jones (Eds.), The Structure, Dynamics and Equilibrium Properties of Colloidal Systems, Kluwer, Dordrecht, 1990.

[9] S.S. Dukhin, J. Lyklema, Langmuir 3 (1987) 94.

[10] J.P. Hsu, Y.C. Kuo, J. Chem. Soc., Faraday Trans. 91 (1995) 4093.

[11] S.Yu. Shulepov, S.S. Dukhin, J. Lyklema, J. Colloid Interface Sci. 171 (1995) 340.

[12] Z. Mandralis, J.H. Wernet, D.L. Feke, J. Colloid Interface Sci. 182 (1996) 26.

[13] J.W. Krozel, J. Colloid Interface Sci. 163 (1994) 437.

[14] J.P. Hsu, Y.C. Kuo, S.J. Tseng, J. Colloid Interface Sci. 195 (1997) 388.

[15] J. Koetz, H. Koepke, G. Schmidt-Naake, P. Zarras, O. Vogl, Polymer 37 (1996) 2275.
[16] J.P. Hsu, Y.C. Kuo, Y.I. Chang, Colloid Polym. Sci. 272 (1994) 946.

[17] J.P. Hsu, Y.C. Kuo, J. Colloid Interface Sci. 171 (1995) 254.

[18] H.P. Wang, J. Jun, L. Blum, Colloid Polym. Sci. 273 (1995) 359.

[19] R.J. Hunter, Foundations of Colloid Science, vol. I, Oxford University Press, London, 1989.

[20] J.P. Hsu, Y.C. Kuo, J. Chem. Phys. 103 (1995) 465.

[21] J. Happel, H. Brenner, Low Reynolds Number Hydrodynamics, Martinus Nijhoff, Boston, MA, 1983.

[22] Y.C. Kuo, J.P. Hsu, Colloid Polym. Sci. 276 (1998) 213.

[23] H.S. Fogler, Elements of Chemical Reaction Engineering, Prentice-Hall, Englewood Cliffs, NJ, 1986.

[24] J.P. Hsu, Y.C. Kuo, Colloid Polym. Sci. 273 (1995) 881.

[25] P.C. Hiemenz, Principles of Colloid and Surface Chemistry, 2nd edn., Dekker, New York, 1986. 